\title{
Photoacoustic imaging in evaluating early intestinal ischemia injury and reperfusion injury in rat models
}

\author{
Rui Wang ${ }^{1 \#}$, Teng Pan ${ }^{2 \#}$, Lin Huang ${ }^{2}$, Chengde Liao ${ }^{1}$, Qinqing Li ${ }^{1}$, Huabei Jiang ${ }^{3}$ Jun Yang ${ }^{1}$ \\ ${ }^{1}$ Department of Radiology, The Third Affiliated Hospital of Kunming Medical University, Yunnan Cancer Hospital/Center, Kunming, China; \\ ${ }^{2}$ School of Electronic Science and Engineering, Center for Information in Medicine, University of Electronic Science and Technology, Chengdu, \\ China; ${ }^{3}$ Department of Medical Engineering, University of South Florida, Tampa, FL, USA
}

\#These authors contributed equally to this work.

Correspondence to: Jun Yang. Department of Radiology, The Third Affiliated Hospital of Kunming Medical University, Yunnan Cancer Hospital/ Center, Kunming 650118, China. Email: imdyang@qq.com; Huabei Jiang. Department of Medical Engineering, University of South Florida, Tampa, FL, USA. Email: hjiang1@usf.edu.

Background: It remains a challenge to distinguish whether the damaged intestine is viable in treating acute mesenteric ischemia. In this study, photoacoustic imaging (PAI) was used to observe intestinal tissue viability after ischemia and reperfusion injury in rats.

Methods: An in vivo study was conducted using forty male SD rats, which were randomly divided into a sham-operated (SO) group, a $1 \mathrm{~h}$ ischemia group, a $2 \mathrm{~h}$ ischemia group, and an ischemia-reperfusion (I/R) group with 10 rats in each group. In the ischemia group, the superior mesenteric artery (SMA) was isolated and clamped for 1 and $2 \mathrm{~h}$, respectively, and in the I/R group, after ischemia for $1 \mathrm{~h}$, the clamp was removed and reperfused for $1 \mathrm{~h}$. The same time interval was used in the SO group. Immediately after establishing the animal model, a PAI examination was performed, and the small intestine was collected for histopathology.

Results: The levels of PAI parameters Hb, HbR, MAP 760, and MAP 840 were increased to different degrees in the ischemia groups, especially in the $2 \mathrm{~h}$ ischemia group, compared with the SO group $(\mathrm{P}<0.05)$, and with prolongation of the ischemia time, the injury was aggravated. All PAI signal levels except $\mathrm{HbO}$ in the I/R group were higher than those in the control group, and the increased range differed, especially in $\mathrm{Hb}$ and MAP 840. Using western blot, compared with the SO group, the BAX increased significantly in the $2 \mathrm{~h}$ ischemia group $(\mathrm{P}<0.05)$, and Caspase- 3 in the experimental group was significantly higher than in the SO group $(\mathrm{P}<0.05)$. The level of HIF-1 $\alpha$ increased in the $2 \mathrm{~h}$ ischemia group and I/R group $(\mathrm{P}<0.05)$, and TUNEL staining showed that the number of positive apoptotic nuclei in the $2 \mathrm{~h}$ ischemia group was significantly higher than in the SO group $(\mathrm{P}<0.05)$. Hematoxylin-eosin $(\mathrm{HE})$ staining showed that ischemia for 2 hours was the most serious, with obvious mucosal damage, extensive epithelial injury, and bleeding.

Conclusions: PAI can be used as an effective tool to detect acute intestinal ischemia injury and quantitatively evaluate tissue viability.

Keywords: Photoacoustic imaging (PAI); acute mesenteric ischemia; intestinal tissue viability; ischemia injury

Submitted Oct 15, 2020. Accepted for publication Feb 14, 2021.

doi: $10.21037 /$ qims-20-1160

View this article at: http://dx.doi.org/10.21037/qims-20-1160 


\section{Introduction}

Intestinal ischemia injury and reperfusion injury have high morbidity and mortality (1) and may occur in many diseases, such as arterial embolism, strangulated hernia, colon cancer, volvulus, toxemia, mesenteric dysfunction, and hypovolemic shock (2). After ischemia injury, the intestinal mucosa is characterized by excessive cell death and loss of barrier function (3), and patients may rapidly decompensate and progress to sepsis and multiple system organ failure (4).

The early identification of damaged tissues before a serious intestinal injury occurs is crucial to implementing the treatment plan (5). While it is particularly important to accurately determine the ischemic area's extent to avoid over-resection of the ischemic small intestine, current assessment methods to determine tissue viability are unreliable. Often this has relied upon the expertise of surgical staff in assessing the pulse and color of the intestine to estimate tissue perfusion and oxygen saturation (6). Also, while conventional computed tomography (CT) and magnetic resonance imaging (MRI) are used to diagnose intestinal ischemia injury, they cannot evaluate changes in hemoglobin concentration and blood oxygen saturation in tissue. Therefore, it is necessary to establish a morphological diagnosis by CT and MRI and further evaluate the status of intestinal blood flow $(7,8)$ by other means. Several commonly used detection techniques, such as sidestream dark-field imaging (9), spectrophotometry, and laser Doppler blood flow measurement, have been used to study intestinal microcirculation (10). However, these technologies, too, cannot provide effective information for judging intestinal activity.

Since there is no effective method to assess the extent of the ischemic injury, which achieves the reliability and repeatability required, we proposed to apply photoacoustic imaging (PAI) to address this problem. PAI is a hybrid imaging method that combines optical contrast and ultrasonic resolution $(11,12)$. It combines high-resolution volume imaging of tissue depths from a few millimeters to centimeters with the ability to analyze drugs with molecular specificity and vascular system and physiological parameters (13). PAI is particularly suitable for vascular imaging because the high concentration of hemoglobin in the blood can achieve strong absorption and produce high contrast with surrounding tissues $(14,15)$. It can also output quantitative data such as hemoglobin concentration and blood oxygen saturation, which indirectly reflect microcirculatory blood flow changes. These unique functions provide a theoretical basis for gastrointestinal PAI. It is important to accurately evaluate the changes to gastrointestinal anatomy and function using the multi wavelength method (16), and at present, the application of PAI technology to evaluate intestinal ischemia injury and reperfusion injury has not been fully studied.

In this study, we hypothesized that PAI could be used to evaluate intestinal ischemia injury and reperfusion injury at an early stage. We explored the characteristics of photoacoustic (PA) signal changes in a rat model of acute mesenteric ischemia injury and reperfusion injury by PAI and histopathological analysis.

\section{Methods}

\section{Animals}

Adult male Sprague Dawley (SD) rats weighing 180-200 g were used and fed in a temperature-controlled room $\left(20 \pm 2{ }^{\circ} \mathrm{C}\right)$ for 12 hours in light and dark circulation. The animals were fasted for 12 hours before PAI but drank water freely.

\section{Experimental protocols}

We randomly divided $40 \mathrm{SD}$ rats into a sham-operation (SO) group, a $1 \mathrm{~h}$ ischemia group, a $2 \mathrm{~h}$ ischemia group, and an ischemia-reperfusion (I/R) group, each numbering 10 rats. Anesthesia was performed by intraperitoneal injection of $10 \%$ chloral hydrate $(1.2 \mathrm{~g} / \mathrm{kg})$, followed by a midline laparotomy to obtain abdominal exposure. After identifying the small intestine and cecum, the superior mesenteric artery (SMA) was carefully exposed, and its root was separated and occluded with noninvasive microvascular forceps. A gauze pad was placed on the intestinal tract and often wetted with warm saline water. After $1 \mathrm{~h}$ of ischemia, the clamp was removed and reperfused for $1 \mathrm{~h}$ to establish the I/R model of SMA. In the ischemia group, the SMA was separated and clamped for 1 and $2 \mathrm{~h}$ and no longer perfused. The SO group was used as a control group, in which the SMA was separated but not clamped after surgery and compared with the corresponding I/R group. Before PAI examination, the intestines were placed on the imaging cavity's surface and smoothed with a cotton swab. The stirred ultrasound agent was then poured into the cavity and smoothed on the surface of the couplants with a cotton swab until it was flush with the probe surface. Multispectral PAI 


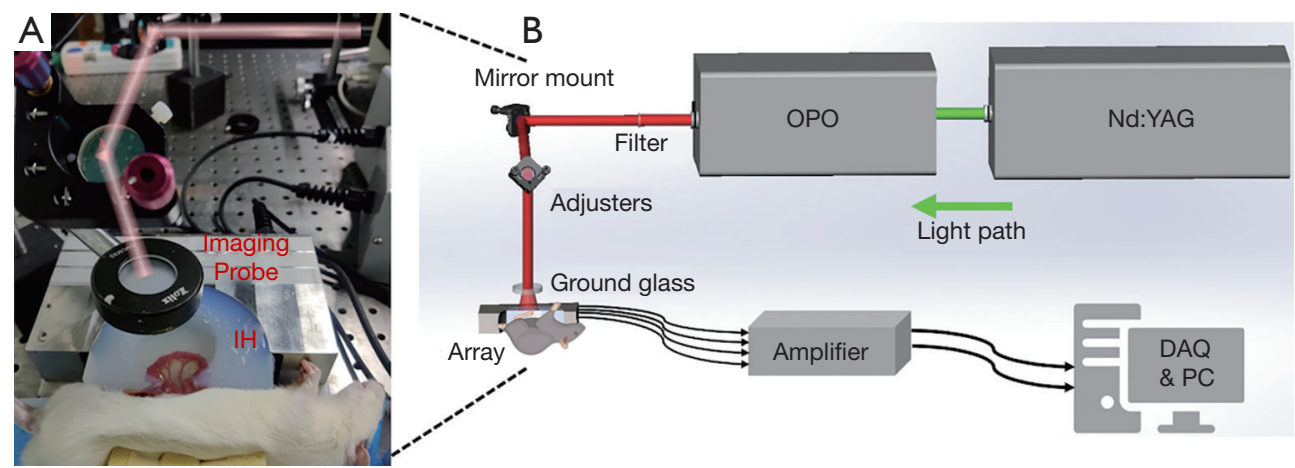

Figure 1 Schematic of the PAI system. (A) the intestines were placed on the holder made by agar power, which closely fitted into the concave surface of the imaging probe; (B) a Nd:YAG-pumped OPO system was used to generate near-infrared puled light which was reflected via two mirrors to the sample for illumination. The stimulated PA signal was amplified and then digitized by the DAQ system. PAI, photoacoustic imaging; OPO, optical parametric oscillator; PA, photoacoustic; DAQ, data acquisition; IH, intestinal holder.

images (760 and $840 \mathrm{~nm}$ ) of each sample were then obtained to resolve the differences in absorption spectra between oxyhemoglobin and deoxyhemoglobin. For each image, the PA signals from a single wavelength were averaged across 10 times to improve the signal-to-noise ratio.

\section{PAI system}

The experimental system for the dynamic monitoring of intestinal ischemia injury and reperfusion injury is shown in Figure 1. A Q-switched Nd:YAG laser using 4 ns pulse duration at $20 \mathrm{~Hz}$ repetition rate (Surelite, Continuum, CA, USA) emits light with a wavelength of $532 \mathrm{~nm}$, which is transformed into 700-960 $\mathrm{nm}$ after passing through an optical parametric oscillator (OPO). After the output laser passes through the two mirrors, the horizontal light path relative to the ground is adjusted to be vertically downward. Before irradiating the biological tissue, the light passes through a ground glass to increase the imaging area, and in the process of collecting the signal, the ground glass is removed, and the light directly illuminates on the tissue. In our study, the incident fluence at $760 \mathrm{~nm}$ was estimated to be $5 \mathrm{~mJ} / \mathrm{cm}^{2}$, which is below the ANSI safety level. A 128-element concave ultrasound transducer array with a central frequency of $5 \mathrm{MHz}$ (Japan Probe Co., Ltd., Japan) was used to receive PA signals. A custom-built 128 -channel preamplifier $(60 \mathrm{~dB})$ was then connected to the probe, and the amplified PA signals were transferred to a 64-channel analog-to-digital system (PXIe5105; NI. Inc.) after 2:1 multiplexing. The developed system's lateral resolution could reach $130 \mu \mathrm{m}$, and the temporal resolution was up to $20 \mathrm{~Hz}$, which is limited by laser re-frequency.

\section{Image reconstruction and data processing}

The delay-and-sum algorithm reconstructed the images. The PA signal intensity was directly related to the absorption of NIR light by tissues, among which hemoglobin and melanin was the main absorption substances. The concentrations of the oxy- and deoxyhemoglobin were unmixed using the following equations:

$$
\left[\begin{array}{l}
C_{H b O} \\
C_{H b R}
\end{array}\right]=\left[\begin{array}{ll}
\varepsilon_{\lambda 1 . H b O} & \varepsilon_{\lambda 1 . H b R} \\
\varepsilon_{\lambda 2 . H b O} & \varepsilon_{\lambda 2 . H b R}
\end{array}\right]^{-1}\left[\begin{array}{l}
M A P_{\lambda 1} / F_{\lambda 1} \\
M A P_{\lambda 2} / F_{\lambda 2}
\end{array}\right]
$$

In the above equations, MAP represents PA amplitude distribution, $F$ is the optical fluence, $\varepsilon$ is the molar extinction coefficient, and $C$ is concentration. To calculate the relative concentration of $\mathrm{HbO}$ and $\mathrm{HbR}$ accurately, two wavelengths (760 and $840 \mathrm{~nm}$ ) in the $700-960 \mathrm{~nm}$ were selected, at which the difference of $\varepsilon$ value between $\mathrm{HbO}$ and HbR is comparatively large (17). The hemoglobin oxygen saturation $\left(\mathrm{SO}_{2}\right)$ was calculated as follows: $\mathrm{SO}_{2}=$ $\mathrm{HbO} /(\mathrm{HbO}+\mathrm{HbR})(18)$.

To define the region of interest (ROI), PAI signal pixel values greater than 0.6 times the maximum signal were selected. As shown in Figure $2 A, B, C, D, E$ inserts, the ROI was exactly the part of the mesenteric blood vessels where the blood perfusion change was obvious during ligation and reperfusion. After ROI was determined, dual-wavelength image signals were used to calculate total hemoglobin and deoxidized and oxyhemoglobin content. 

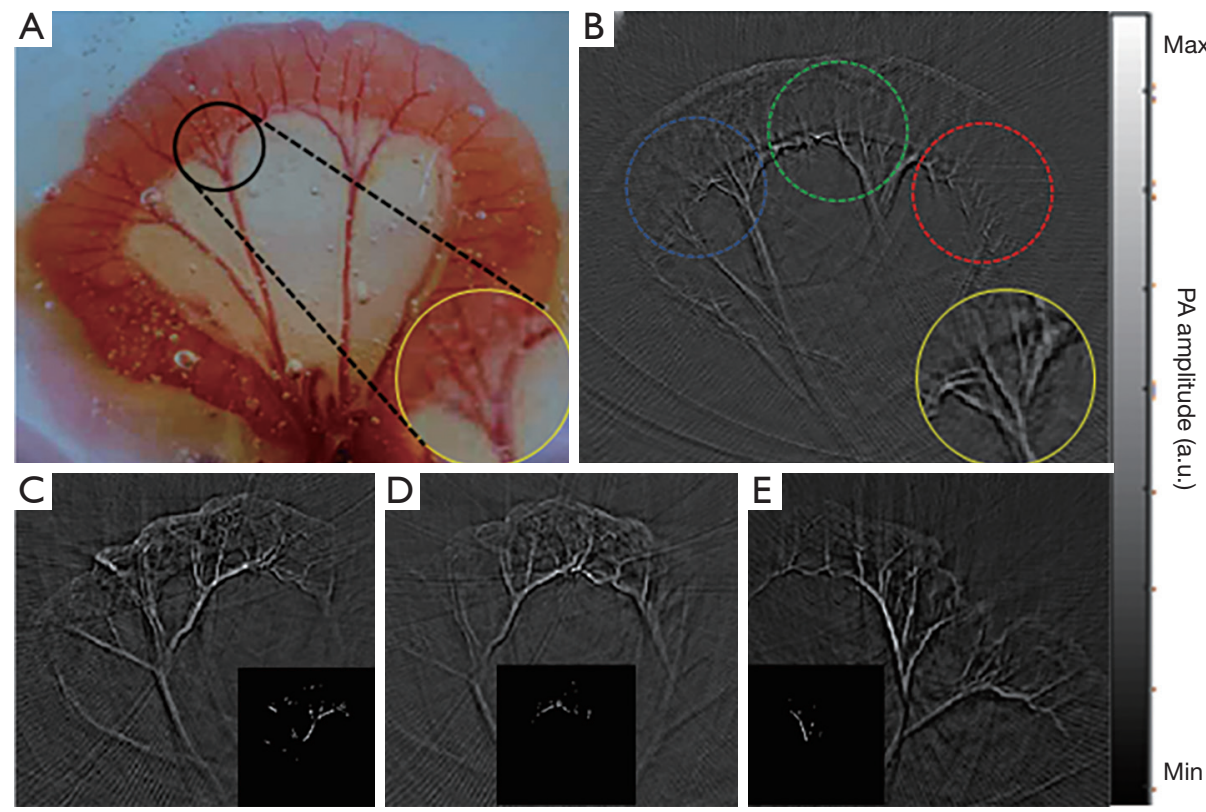

Figure 2 Intestinal images of a rat. (A) Intestinal photograph; (B) corresponding PA image. The color circles represent different regions of interest, and yellow circle represents the partially enlarged part; $(\mathrm{C}, \mathrm{D}, \mathrm{E})$ show the PAI results of the same intestinal tissue at three different lighting areas respectively, with the insets showing the regions of interest where the PA signal was significant. PA, photoacoustic; PAI, photoacoustic imaging.

\section{Histopathology}

\section{Hematoxylin-eosin (HE)}

After PAI, the animals were killed, $100 \mathrm{~mL}$ normal saline containing heparin was perfused through the heart, and $100 \mathrm{~mL} \mathrm{4 \%} \mathrm{paraformaldehyde} \mathrm{was} \mathrm{injected.} \mathrm{The} \mathrm{tissue}$ samples were then fixed in $10 \%$ formalin solution and dehydrated overnight in 30\% sucrose. Embedded in paraffin, $5 \mu \mathrm{m}$ slices were cut by a slicing machine then dried overnight in an incubator at $45{ }^{\circ} \mathrm{C}$. The sections were further stained with $\mathrm{HE}$ and observed under light microscope.

\section{Western blot}

As mentioned previously, the small intestinal tissue was scraped and homogenized in a 10 -fold volume of cold RIPA buffer. After incubation on ice for 30 minutes, the cell lysate was centrifuged at 14,000 rpm for 15 minutes, and the protein concentration in the supernatant was determined. The supernatant was dissolved in a $2 \times$ electrophoresis sample buffer at a ratio of $1: 1$, subjected to high-temperature heat sealing, and stored at $-20{ }^{\circ} \mathrm{C}$ for use. The extracted proteins were then separated by twelve alkyl sulfate polyacrylamide gel electrophoresis with different concentrations, and the separated proteins were electrotransferred to polyvinylidene fluoride two membranes. This was sealed with $5 \%$ non-fat milk, incubated overnight with primary antibody at $4{ }^{\circ} \mathrm{C}$, and then incubated with horseradish peroxidase-labeled secondary antibody at room temperature for $1 \mathrm{~h}$. Specific proteins including Cleaved Caspase-3, Bcl-2, BAX, and HIF-1 $\alpha$ were observed with enhanced chemiluminescence enhancement kits. The following antibodies were used: rabbit antiCleaved Caspase-3 (\#9664, Cell Signaling Technology), anti-Bcl-2 antibody (ab59348, Abcam), BAX Antibody (\#2772, Cell Signaling Technology), rabbit, and anti-HIF$1 \alpha$ (\#14179, Cell Signaling Technology). The band density was observed by a gel electrophoresis imaging system and quantified by ImageJ software (https://imagej.nih.gov/).

\section{TUNEL staining}

TUNEL method was used to identify apoptotic cells in tissue sections of double-stranded DNA fragments, which were labeled by immobilized enzyme nucleotides. We used the apoptosis detection kit (in situ cell death detection kitPOD, Roche). The sample was rehydrated in alcohol then 
immersed in phosphate-buffered saline (PBS) at room temperature for 5 minutes. The tissues were then treated with a cell permeabilizer $(0.1 \%$ sodium citrate $+0.1 \%$ Triton $\mathrm{X}-100)$ for 8 minutes and washed three times with PBS at 21-37 ${ }^{\circ} \mathrm{C}$. The TUNEL reaction mixture was prepared, and the slide was then immersed in $1 \times$ TDT labeled buffer for 5 minutes. After stopping the labeling reaction, the samples were covered with diluted anti-BrdU and cultured at $37^{\circ} \mathrm{C}$ for 1 hour; then, each slice was incubated with $50 \mu \mathrm{L}$ DAPI at room temperature for 10 minutes and photographed under a fluorescence microscope.

\section{Statistical analysis}

All data were expressed as mean \pm standard error unless otherwise stated. A one-way ANOVA test was used to compare different groups. SPSS 23.0 software (SPSS, Inc., Chicago, IL, USA) was used for statistical analysis, and after statistics, a $\mathrm{P}$ value less than 0.05 was considered to have statistical significance.

\section{Results}

\section{The signal intensity of PAI in intestine injury}

After light passed through the ground glass, the irradiated area became larger, and most of the tissue of the intestine in the phantom cavity could be observed. Figure 2 shows one of the PA images of an SD rat's intestines in the control group using the PAI system. Figure $2 A$ is the rat intestinal tissue image, and Figure $2 B$ is the corresponding $\mathrm{PA}$ reconstruction image. As shown in the PA image, the intestine border is outlined, and several mesenteric blood vessels and even capillaries are clearly visible. The area in the middle of the image is significantly brighter than the tissue at the edges due to uneven illumination.

When the ground glass was removed, the light was irradiated on the tissue with more concentrated energy and a smaller spot, but at the cost of reducing the imaging area. The light spot's position could be moved two-dimensionally by the adjusters on the mirror mount so that the light could irradiate different intestinal areas of interest. As shown in the dotted line circles in Figure 2B, the left and right edges and the middle area are usually selected to realize the signal collection of multiple samples of the same intestine.

PAI parameters of each group are shown in Figure 3. While there are no significant changes in the normal small intestine's PAI signal parameters, the PAI signal in the experimental group is almost stronger than in the SO group. In general, the levels of $\mathrm{Hb}, \mathrm{HbR}, \mathrm{MAP} 760$, and MAP 840 in the ischemia group increased with varying degrees, especially in the $2 \mathrm{~h}$ ischemia group $(\mathrm{P}<0.05)$, and with the prolongation of ischemia time, the injury was aggravated. The levels of $\mathrm{Hb}$ and MAP 840 at $1 \mathrm{~h}$ after ischemia were higher than those in the SO group, but the difference was not statistically significant $(\mathrm{P}>0.05)$. Except for $\mathrm{HbO}$, the PAI signal level in the I/R group was higher than that in the control group, and the range of increase was different, and the increase was most obvious in $\mathrm{Hb}$ and MAP $840(\mathrm{P}<0.05)$. There was no significant difference in $\mathrm{HbO}$ and $\mathrm{SO}_{2}$ between experimental and control groups, and compared with the SO group, all PAI signal levels increased except HbO. Among them, it increased 1.011.50 times in the $1 \mathrm{~h}$ ischemia group, $1.30-1.59$-fold in the $2 \mathrm{~h}$ ischemia group, and 1.01-1.38-fold in the I/R group.

\section{HE staining}

HE staining of small intestine sections is shown in Figure 4. In the SO group, the small intestine villi were orderly arranged, the cell size and extracellular space were consistent, and there was no obvious inflammatory cell infiltration. Swelling and congestion of intestinal villi and exfoliation of epithelial cells were found in the $1 \mathrm{~h}$ and $2 \mathrm{~h}$ ischemia injury groups. Also, obvious mucosal injury, extensive epithelial injury, lamina propria disintegration, and hemorrhage were observed in the $2 \mathrm{~h}$ ischemia group.

\section{Ischemia induced Caspase-3, BAX, Bcl-2, and HIF-1 $\alpha$ expression in intestines}

Compared with the SO group, the expression of Cleaved Caspase- 3 in the ischemia group and the reperfusion group continued to increase. The Cleaved Caspase- 3 in the $2 \mathrm{~h}$ ischemia group and I/R group was significantly higher than the baseline value $(\mathrm{P}<0.05)$. BAX increased in the experimental group and the $1 \mathrm{~h}$ ischemia group compared with the SO group $(\mathrm{P}>0.05)$, but was significantly increased in the $2 \mathrm{~h}$ ischemia group $(\mathrm{P}<0.05)$. The expression levels of Bcl-2 in the ischemia group and the I/R group were lower than those in the SO group, but the difference was not statistically significant $(\mathrm{P}>0.05)$. The expression of HIF- $1 \alpha$ increased gradually in the experimental group, and compared with the SO group; the HIF- $1 \alpha$ level was increased in the $2 \mathrm{~h}$ ischemia group and $\mathrm{I} / \mathrm{R}$ group $(\mathrm{P}<0.05)$ (Figure 5). 

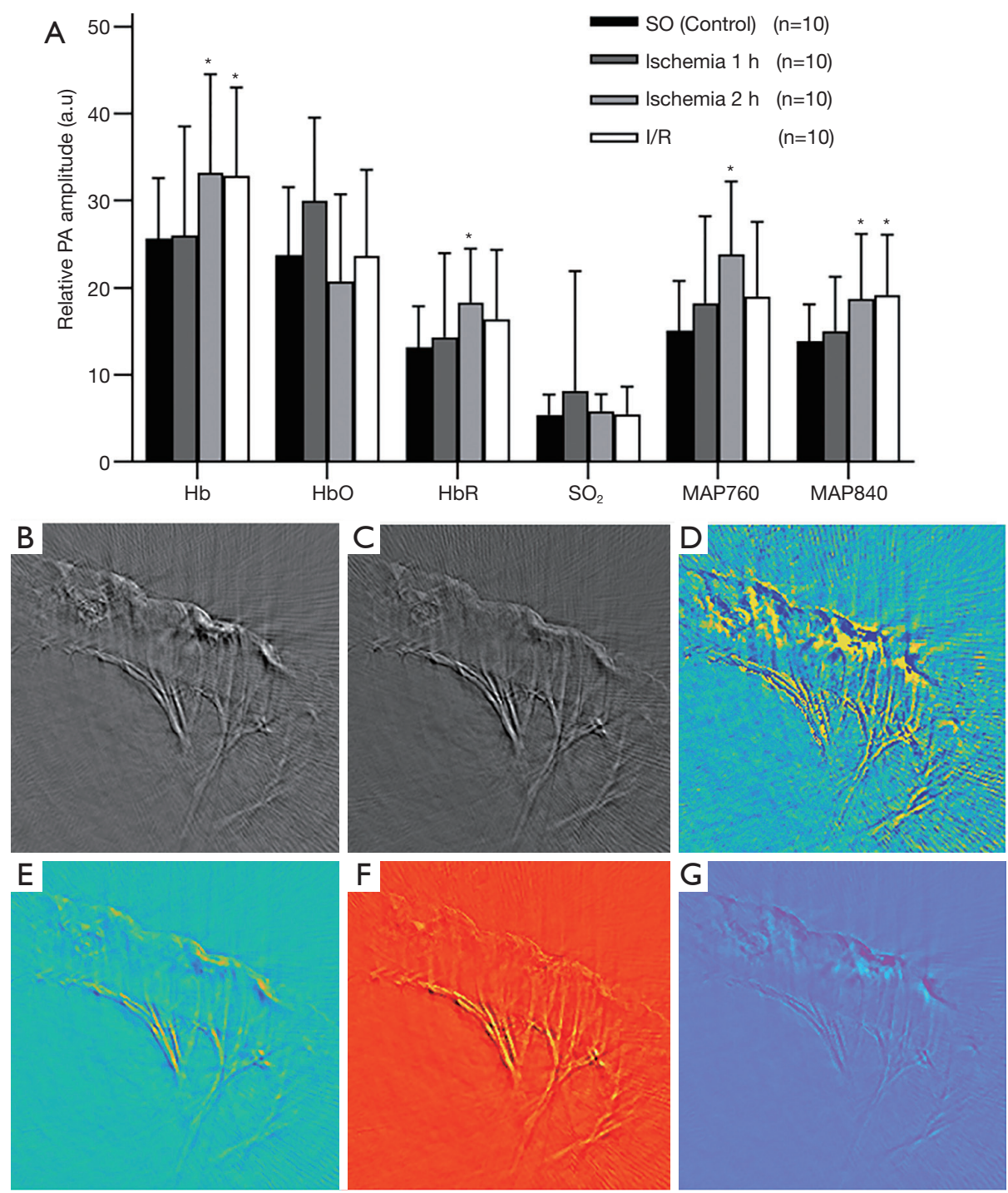

Figure 3 Signal strength of PAI parameters. (A) The longitudinal development of six parameters from PAI. Compared with the control group, the signal intensities of $\mathrm{Hb}, \mathrm{HbR}, \mathrm{SO}_{2}, \mathrm{MAP} 760$, and MAP 840 in the ischemia group and the I/R group were increased in varying degrees, especially in the $2 \mathrm{~h}$ ischemia group; (B,C,D,E,F,G) are the parameter diagram of MAP 760, MAP 840, SO $, \mathrm{Hb}, \mathrm{HbO}$, and $\mathrm{HbR}$, respectively. (* indicates compared with control $\mathrm{P}<0.05$ ). PAI, photoacoustic imaging; PA, photoacoustic; SO, sham-operated; I/R, ischemiareperfusion.

\section{TUNEL staining}

TUNEL staining was used to identify apoptotic nuclei induced by ischemia and reperfusion. In the SO group, a small number of TUNEL positive nuclei were found in the intestine, and the ischemia injury induced by SMA hemostatic clip increased the number of TUNEL positive nuclei. Also, the number of TUNEL positive nuclei in the $2 \mathrm{~h}$ ischemia group was significantly higher than in the SO group $(\mathrm{P}=0.03)$. The TUNEL staining of the intestine is shown in Figure 6.

\section{Discussion}

The evaluation of intestinal tissue viability is key to the treatment of acute mesenteric ischemia. Surgeons are often faced with a very difficult choice, as extensive intestinal resection may cause serious complications such 

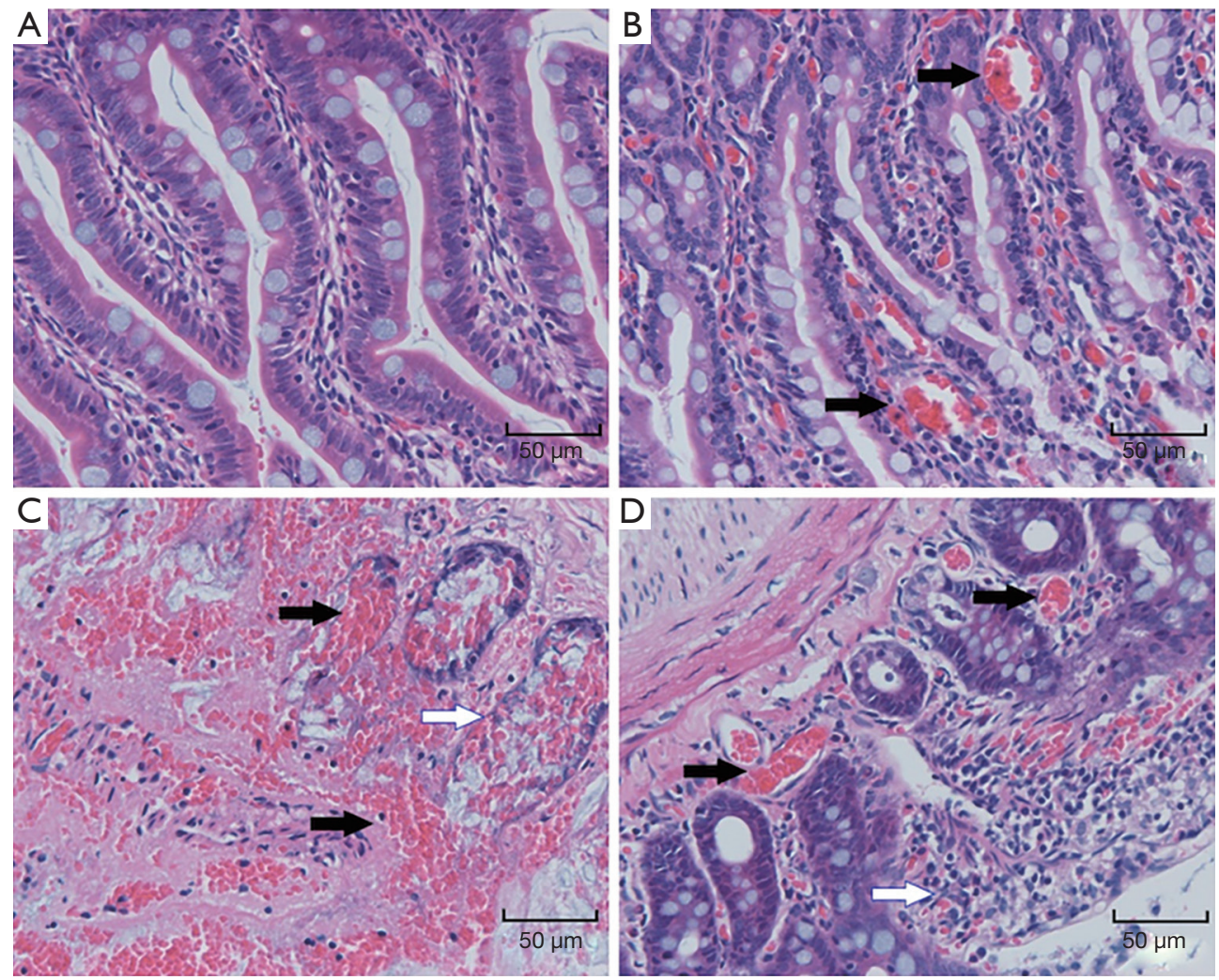

Figure 4 Histopathological changes of intestinal mucosa under light microscope (magnification, $\times 40$; HE staining). (A) SO group (control group); (B) in the $1 \mathrm{~h}$ ischemia group, red blood cells were shed from some capillaries; (C) in the $2 \mathrm{~h}$ ischemia group the epithelium of intestinal mucosa was exfoliated, and the villi were dissolved and damaged; (D) in the I/R group the epithelium of some small intestinal mucosa exfoliated and damaged crypt could be seen. The black arrow indicates bleeding and the white arrow indicates necrosis and dissolution of intestinal villi. HE, hematoxylin-eosin; SO, sham-operated; I/R, ischemia-reperfusion.
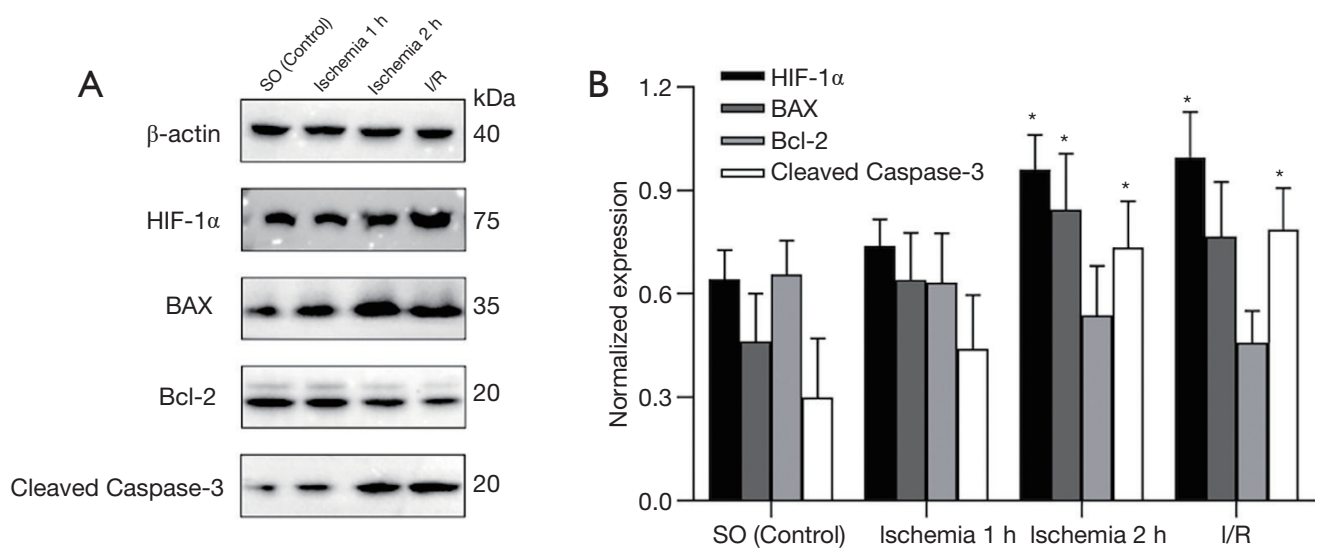

Figure 5 Expression of HIF-1 $\alpha$, BAX, Bcl-2, and Cleaved Caspase-3 in the intestinal mucosa of rats with intestinal I/R injury. (A) the level of Cleaved Caspase- 3 in the $2 \mathrm{~h}$ ischemia group and the I/R group was significantly higher than that in the control group, and the BAX level in the $2 \mathrm{~h}$ ischemia group was significantly higher than that in the control group. The level of HIF-1 $\alpha$ increased in the $2 \mathrm{~h}$ ischemia group and the I/R group; (B) the histogram after quantitative analysis by Image J software. (* indicates compared with control $\mathrm{P}<0.05$ ). I/R, ischemia-reperfusion; $\mathrm{SO}$, sham-operated. 

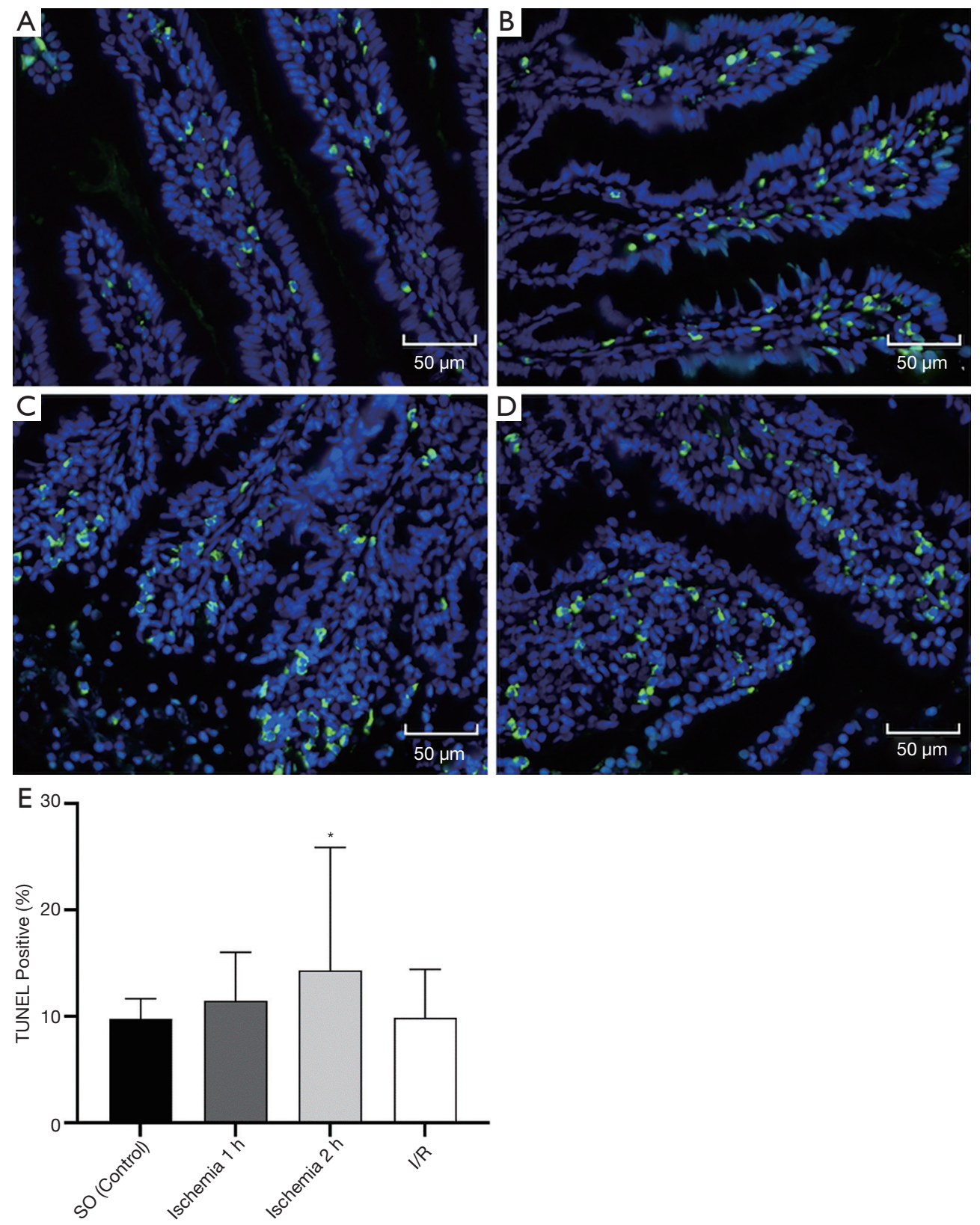

Figure 6 TUNEL labeling of apoptotic nuclei (magnification, $\times 40$ ). (A) SO group (control group); (B) 1 h ischemia group; (C) 2 h ischemia group; (D) I/R group. The apoptosis of intestinal mucosal cells was the most serious in the $2 \mathrm{~h}$ ischemia group; (E) quantitative results of positive TUNEL staining in each group. ( ${ }^{*}$ indicates compared with control $\mathrm{P}<0.05$ ). SO, sham-operated; I/R, ischemia-reperfusion.

as short bowel syndrome (19). However, if the necrotic part of the intestinal resection is not sufficient, a severe systemic inflammatory response may occur, which may induce multiple organ dysfunction (20). Therefore, the accurate judgment of the range of the involved bowel is very important for the operation's success. In recent years, fluorescein, Doppler, electromyography, and radionuclide have been used to determine intestinal viability (21-23), but the complex operation method and high detection cost limit their wide application. CT and MRI are also used to predict or evaluate intestinal viability and have certain diagnostic value for intestinal ischemia injury $(24,25)$. 
However, these imaging techniques usually produce motion artifacts due to the patient's heartbeat and respiration (26), and, most importantly, these devices cannot be used during surgery. In contrast, PAI is an objective evaluation method and can be used for quantitative analysis to reflect changes to pathophysiology and can be used for real-time imaging during surgery.

Compared with conventionally used clinical evaluation methods for intestinal ischemia and reperfusion, the method used in this study was not affected by motion artifacts, and the tissue thickness was up to several millimeters. PAI is a non-invasive medical imaging method that combines the high contrast characteristics of pure optical imaging and the high penetration depth characteristics of pure ultrasound imaging and can also provide high resolution and high contrast tissue imaging. In our experiment, the mesenteric vessels in which we were interested were only a few millimeters below the surface of the irradiated tissue, and under proper illumination, the tissue depth could even be within $5 \mathrm{~cm}$ (depending on the illuminated organs) (27). When using a single acquisition mode, PAI can be considered instantaneous (usually completed in tens of milliseconds), which means no motion artifacts are generated $(28,29)$. With this method, we can indirectly obtain quantitative information on the degree of ischemia and blood reperfusion of small intestine vessels, with a resolution of up to $0.1 \mathrm{~mm}$.

We used histopathological results to verify whether PAI in intestinal tissue can assess tissue viability. As described, PAI detected changes in intestinal tissue viability associated with intestinal ischemia time in a model of acute mesenteric ischemia injury. It is noteworthy that the small intestine's signal intensity in the $2 \mathrm{~h}$ ischemia group was significantly increased to $1.30-1.59$ times of that in the control group. PAI indirectly showed that the blood circulation near the injured intestine was much higher than that in the normal intestine, as manifested by acute inflammation. Villi edema, epithelial cells shedding, tissue edema, congestion, and bleeding were observed in the ischemia group, blood vessels and lymphatic vessels were obviously dilated, and glands were damaged. The results showed that intestinal ischemia could significantly increase the apoptosis of intestinal mucosal cells, and with the increase of intestinal ischemia time, apoptosis also increased and was most serious in the $2 \mathrm{~h}$ ischemia group. Using Western blot, we found that the expression of Bcl2 was significantly decreased in the experimental group, but the expression of BAX was increased and highest in the $2 \mathrm{~h}$ ischemia group. Bcl-2 and BAX belong to a family, which regulates apoptosis by controlling the mitochondrial membrane's permeability (30). The decrease of Bcl-2 levels and increase of BAX levels indicates that cells' resistance to apoptosis is weakened. We found that intestinal ischemia can increase the apoptosis of intestinal epithelial cells, which was manifested by an increase in the apoptosis index and Caspase- 3 protein expression. In villous epithelium, Caspase- 3 activation accompanied by the downregulation of Bcl-2 expression seems to be related to apoptosis induction. TUNEL staining is a combination of molecular biology and morphology, and in the experimental group, we observed that TUNEL staining showed that apoptosis was most serious in the $2 \mathrm{~h}$ ischemia group.

In addition to observing the indexes of apoptosis, we also observed the indexes related to hypoxia status. The increased expression of HIF-1 $\alpha$ indicates severe hypoxia and reflects tissue hypoxia (31). We found that the HIF-1 $\alpha$ level was significantly increased in the $2 \mathrm{~h}$ ischemia group, indicating that hypoxia was severe in this group. Simultaneously, the levels of $\mathrm{Hb}, \mathrm{HbR}$, and MAP 760 in this group were significantly higher than those in the control group. PA parameters directly indicate the change of oxygen content in tissues (32). Nasiriavanaki et al. (33) used a PA tomography system to observe brain functional connectivity by measuring hemoglobin concentration in the mouse brain and found that while hyperoxia enhanced their functional connectivity, hypoxia weakened it. Histopathological examination of the tissue samples in our study showed that PAI could quickly, and real-time assess the degree of intestinal ischemia injury in the experimental model.

In the process of ischemia and reperfusion, there is a dependence between reperfusion injury and ischemia time. The ischemia process itself can cause injury, which is also the basis of reperfusion injury $(34,35)$. The essence of reperfusion injury is to further aggravate or convert the injury in the ischemic phase into irreversible injury after blood flow recovery (36). Therefore, the most important factor affecting reperfusion injury is ischemia time (37). While all organs can tolerate a degree of ischemia if the time is short and there is no obvious reperfusion injury after blood supply is restored, if the ischemia time is long, the recovery of blood supply can easily lead to reperfusion injury. In our experimental group, there was no significant difference in PAI between the $1 \mathrm{~h}$ ischemia group and the SO group, suggesting that intestinal ischemia may be reversible at this time. However, our experiment focused on the early detection of intestinal I/R injury rather than 
monitoring the pathological process of $\mathrm{I} / \mathrm{R}$ injury, so we did not conduct a 2-hour reperfusion injury study.

In a previous experimental I/R model, the observation of intestinal viability loss was limited to prolonged ischemia $(38,39)$. An in vitro monitoring of intestinal permeability showed that it took at least 60 minutes to detect a change in intestinal barrier function after ischemic injury, which could not quickly judge the change to intestinal viability (3). In our study, PAI could detect intestinal viability after as little as 2 hours of ischemic intestinal injury and after as little as 1 hour of I/R injury, demonstrating PAI can quickly assess intestinal damage in real-time. The strong light absorption of hemoglobin in tissues gives blood vessels very high contrast in PA images, which is the basis for PAI technology to detect hemoglobin content (40). The light absorption detected by PAI results from a complex interaction between blood volume and oxygen consumption. Ischemia usually reduces part of the intestinal blood volume and oxygenation, leading to a decrease in tissue absorption characteristics (41). Spectral analysis of PAI images showed that there were significant differences in PAI images between the experimental models with different ischemic times, and PAI could judge the degree of ischemic injury. In addition to the early diagnosis of intestinal ischemia, PAI may provide helpful information for intestinal ischemia injury treatment.

Our research has some limitations. Firstly, in the acute intestinal ischemia stage, inflammatory cells and inflammatory cytokines were activated and released, and those may be related to PAI, but we did not focus on change to inflammatory markers. Secondly, we only studied the early evaluation and diagnosis of ischemic intestinal injury and did not pay attention to evaluating chronic ischemia and assessing efficacy, which will be considered in future work.

\section{Conclusions}

In this study, we used the high resolution and clear field of vision provided by a PAI system to evaluate its application in rats with intestinal ischemia injury rats in real-time. The results showed that PAI could reflect pathophysiological changes to the intestine, such as apoptosis and hypoxia, as a means of evaluating the severity of the ischemic injury. This may be helpful for surgeons to evaluate ischemic injury objectively during living body surgery.

\section{Acknowledgments}

Funding: This study was supported by the National Natural
Science Foundation of China (Grants 82060313 and 81760316).

\section{Footnote}

Conflicts of Interest: All authors have completed the ICMJE uniform disclosure form (available at http://dx.doi. org/10.21037/qims-20-1160). The authors have no conflicts of interest to declare.

Ethical Statement: All animal protocols used in this study were approved by the Animal Ethics Committee of Kunming Medical University. All the experimental protocols and animal handling were conducted in strict accordance with the guidelines of our institutional animal ethics committee.

Open Access Statement: This is an Open Access article distributed in accordance with the Creative Commons Attribution-NonCommercial-NoDerivs 4.0 International License (CC BY-NC-ND 4.0), which permits the noncommercial replication and distribution of the article with the strict proviso that no changes or edits are made and the original work is properly cited (including links to both the formal publication through the relevant DOI and the license). See: https://creativecommons.org/licenses/by-nc-nd/4.0/.

\section{References}

1. Nadatani $Y$, Watanabe T, Shimada S, Otani K, Tanigawa T, Fujiwara Y. Microbiome and intestinal ischemia/ reperfusion injury. J Clin Biochem Nutr 2018;63:26-32.

2. Eryilmaz S, Turkyilmaz Z, Karabulut R, Gulburun MA, Poyraz A, Gulbahar O, Arslan B, Sonmez K. The effects of hydrogen-rich saline solution on intestinal anastomosis performed after intestinal ischemia reperfusion injury. $\mathrm{J}$ Pediatr Surg 2020;55:1574-8.

3. Hsiao JK, Huang CY, Lu YZ, Yang CY, Yu LC. Magnetic resonance imaging detects intestinal barrier dysfunction in a rat model of acute mesenteric ischemia/reperfusion injury. Invest Radiol 2009;44:329-35.

4. Jensen AR, Doster DL, Hunsberger EB, Manning MM, Stokes SM, Barwinska D, March KL, Yoder MC, Markel TA. Human adipose stromal cells increase survival and mesenteric perfusion following intestinal ischemia and reperfusion injury. Shock 2016;46:75-82.

5. Struck R, Wittmann M, Muller S, Meybohm P, Müller A, Bagci S. Effect of remote ischemic preconditioning on 
intestinal ischemia-reperfusion injury in adults undergoing on-pump CABG surgery: a randomized controlled pilot trial. J Cardiothorac Vasc Anesth 2018;32:1243-7.

6. Mariani P, Slim K. Enhanced recovery after gastrointestinal surgery: the scientific background. J Visc Surg 2016;153:S19-25.

7. Shi H, Li R, Qiang J, Li Y, Wang L, Sun R. Computed tomography perfusion imaging detection of microcirculatory dysfunction in small intestinal ischemiareperfusion injury in a porcine model. PLoS One 2016;11:e0160102.

8. Zhao DW, Zhang LT, Cheng HY, Zhang YL, Min JY, Xiao HL, Wang Y. Monitoring dynamic alterations in calcium homeostasis by T1-mapping manganese-enhanced MRI (MEMRI) in the early stage of small intestinal ischemiareperfusion injury. NMR Biomed 2015;28:958-66.

9. Lehmann Ch, Abdo I, Kern H, Maddison L, Pavlovic D, Sharawi N, Starkopf J, Hall R, Johnson P, Williams L, Cerny V; MiDAS (Microcirculation Diagnostics and Applied Studies) group. Clinical evaluation of the intestinal microcirculation using sidestream dark field imaging--recommendations of a round table meeting. Clin Hemorheol Microcirc 2014;57:137-46.

10. Dima A, Gateau J, Claussen J, Wilhelm D, Ntziachristos V. Optoacoustic imaging of blood perfusion: techniques for intraoperative tissue viability assessment. J Biophotonics 2013;6:485-92.

11. Zhang S, Qi L, Li X, Liu J, Huang S, Wu J, Liu R, Feng Y, Feng Q, Chen W. Photoacoustic imaging of living mice enhanced with a low-cost contrast agent. Biomed Opt Express 2019;10:5744-54.

12. Knieling F, Gonzales Menezes J, Claussen J, Schwarz M, Neufert C, Fahlbusch FB, Rath T, Thoma OM, Kramer V, Menchicchi B, Kersten C, Scheibe K, Schürmann S, Carlé B, Rascher W, Neurath MF, Ntziachristos V, Waldner MJ. Raster-scanning optoacoustic mesoscopy for gastrointestinal imaging at high resolution. Gastroenterology 2018;154:807-9.e3.

13. Qin W, Qi W, Xi L. Quantitative investigation of vascular response to mesenteric venous thrombosis using largefield-of-view photoacoustic microscopy. J Biophotonics 2019;12:e201900198.

14. Karlas A, Kallmayer M, Fasoula NA, Liapis E, Bariotakis M, Krönke M, Anastasopoulou M, Reber J, Eckstein HH, Ntziachristos V. Multispectral optoacoustic tomography of muscle perfusion and oxygenation under arterial and venous occlusion: a human pilot study. J Biophotonics 2020;13:e201960169.
15. Bayer CL, Wlodarczyk BJ, Finnell RH, Emelianov SY. Ultrasound-guided spectral photoacoustic imaging of hemoglobin oxygenation during development. Biomed Opt Express 2017;8:757-63.

16. Petrov YY, Petrova IY, Patrikeev IA, Esenaliev RO, Prough DS. Multiwavelength optoacoustic system for noninvasive monitoring of cerebral venous oxygenation: a pilot clinical test in the internal jugular vein. Opt Lett 2006;31:1827-9.

17. Yang J, Zhang G, Wu M, Shang Q, Huang L, Jiang H. Photoacoustic assessment of hemodynamic changes in foot vessels. J Biophotonics 2019;12:e201900004.

18. Yang J, Zhang G, Shang Q, Wu M, Huang L, Jiang H. Detecting hemodynamic changes in the foot vessels of diabetic patients by photoacoustic tomography. J Biophotonics 2020;13:e202000011.

19. Turato WM, Sales-Campos H, Braga CB, Cunha SF, Silvah JH, da Silva JS, Marchini JS, de Barros Cardoso CR. The impact of intestinal resection on the immune function of short bowel syndrome patients. Hum Immunol 2016;77:1202-8.

20. Osuka A, Kusuki H, Matsuura H, Shimizu K, Ogura H, Ueyama M. Acute intestinal damage following severe burn correlates with the development of multiple organ dysfunction syndrome: A prospective cohort study. Burns 2017;43:824-9.

21. Li T, Tomimatsu T, Ito K, Horie T. Fluoresceinmethotrexate transport in brush border membrane vesicles from rat small intestine. Life Sci 2003;73:2631-9.

22. Sugito K, Kusafuka T, Hoshino M, Inoue M, Ikeda T, Hagiwara N, Koshinaga T. Usefulness of color doppler sonography and $99 \mathrm{~m}$ Tc-RBC scintigraphy for preoperative diagnosis of a venous malformation of the small intestine in a 2-year-old child. J Clin Ultrasound 2008;36:56-8.

23. Schiemer JF, Heimann A, Somerlik-Fuchs KH, Ruff R, Hoffmann KP, Baumgart J, Berres M, Lang H, Kneist W. Five-fold gastrointestinal electrical stimulation with electromyography-based activity analysis: towards multilocular theranostic intestinal implants. J Neurogastroenterol Motil 2019;25:461-70.

24. Minhas AS, Sharkey J, Randtke EA, Murray P, Wilm B, Pagel MD, Poptani H. Measuring kidney perfusion, $\mathrm{pH}$, and renal clearance consecutively using MRI and multispectral optoacoustic tomography. Mol Imaging Biol 2020;22:494-503.

25. Tseng CY, Chang CM, Yang SC, Chia-Yu Chang J, Chen JD, Huang HH, How CK, Hung-Tsang Yen D, Huang MS. Spontaneous intramural intestinal hemorrhage versus 
acute mesenteric ischemia by CT evaluation. Intern Med 2016;55:2337-41.

26. Copin P, Zins M, Nuzzo A, Purcell Y, Beranger-Gibert S, Maggiori L, Corcos O, Vilgrain V, Ronot M. Acute mesenteric ischemia: a critical role for the radiologist. Diagn Interv Imaging 2018;99:123-34.

27. Ivankovic I, Deán-Ben XL, Lin HA, Zhang Z, Trautz B, Petry A, Görlach A, Razansky D. Volumetric optoacoustic tomography enables non-invasive in vivo characterization of impaired heart function in hypoxic conditions. Sci Rep 2019;9:8369.

28. Lediju Bell MA. Photoacoustic imaging for surgical guidance: principles, applications, and outlook. J Appl Phys 2020;128:060904.

29. Zhong Y, Zou Y, Liu L, Li R, Xue F, Yi T. pH-responsive Ag2S nanodots loaded with heat shock protein 70 inhibitor for photoacoustic imaging-guided photothermal cancer therapy. Acta Biomater 2020;115:358-70.

30. Anantram A, Degani M. Targeting cancer's Achilles' heel: role of BCL-2 inhibitors in cellular senescence and apoptosis. Future Med Chem 2019;11:2287-312.

31. Semenza GL. Targeting hypoxia-inducible factor 1 to stimulate tissue vascularization. J Investig Med 2016;64:361-3.

32. Karmacharya MB, Sultan LR, Kirkham BM, Brice AK, Wood AKW, Sehgal CM. Photoacoustic imaging for assessing tissue oxygenation changes in rat hepatic fibrosis. Diagnostics (Basel) 2020;10:705.

33. Nasiriavanaki M, Xia J, Wan H, Baue AQ, Culver JP, Wang LV. High-resolution photoacoustic tomography of resting-state functional connectivity in the mouse brain. Proc Natl Acad Sci U S A 2014;111:21-6.

34. Shiva N, Sharma N, Kulkarni YA, Mulay SR, Gaikwad AB. Renal ischemia/reperfusion injury: an insight on in vitro

Cite this article as: Wang R, Pan T, Huang L, Liao C, Li Q, Jiang H, Yang J. Photoacoustic imaging in evaluating early intestinal ischemia injury and reperfusion injury in rat models. Quant Imaging Med Surg 2021;11(7):2968-2979. doi: 10.21037/ qims-20-1160 and in vivo models. Life Sci 2020;256:117860.

35. Sánchez-Hernández CD, Torres-Alarcón LA, González-

Cortés A, Peón AN. Ischemia/reperfusion injury: pathophysiology, current clinical management, and potential preventive approaches. Mediators Inflamm 2020;2020:8405370.

36. Soares ROS, Losada DM, Jordani MC, Évora P, Castro-ESilva O. Ischemia/reperfusion injury revisited: an overview of the latest pharmacological strategies. Int J Mol Sci 2019;20:5034.

37. Schmucker RW, Mendenhall SD, Reichensperger JD, Yang M, Neumeister MW. Defining the salvage time window for the use of ischemic postconditioning in skeletal muscle ischemia reperfusion injury. J Reconstr Microsurg 2015;31:597-606.

38. Zhao L, Luo L, Chen J, Xiao J, Jia W, Xiao Y. Utilization of extracorporeal membrane oxygenation alleviates intestinal ischemia-reperfusion injury in prolonged hemorrhagic shock animal model. Cell Biochem Biophys 2014;70:1733-40.

39. Boettcher M, Eschenburg G, Mietzsch S, JiménezAlcázar M, Klinke M, Vincent D, Tiemann B, Bergholz R, Reinshagen K, Fuchs TA. Therapeutic targeting of extracellular DNA improves the outcome of intestinal ischemic reperfusion injury in neonatal rats. Sci Rep 2017;7:15377.

40. Tzoumas S, Nunes A, Olefir I, Stangl S, Symvoulidis P, Glasl S, Bayer C, Multhoff G, Ntziachristos V. Eigenspectra optoacoustic tomography achieves quantitative blood oxygenation imaging deep in tissues. Nat Commun 2016;7:12121.

41. Li Z, Li H, Chen H, Xie W. In vivo determination of acute myocardial ischemia based on photoacoustic imaging with a focused transducer. J Biomed Opt 2011;16:076011. 